\title{
Shape change as entropic phase transition: A study using Jarzynski relation $^{\#}$
}

\author{
MOUPRIYA DAS, DEBASISH MONDAL and DEB SHANKAR RAY* \\ Department of Physical Chemistry, Indian Association for the Cultivation of Science, Jadavpur, \\ Kolkata 700 032, India \\ e-mail: pcdsr@iacs.res.in
}

\begin{abstract}
A Brownian particle in a confined space with varying cross-section, experiences an effective entropic potential in reduced dimension. We modulate the shape of the confinement and examine the nature of dynamical transition between two distinct thermalized entropic states corresponding to different shapes of the enclosure, using Jarzynski relation on the basis of work-distribution over non-equilibrium paths. Our analysis reveals that modulating the shape of the boundaries of the enclosure makes the resident Brownian particles feel an entropic phase transition.
\end{abstract}

Keywords. Fick-Jacobs equation; entropic potental; Jarzynski equality; phase transition.

\section{Introduction}

Phase transition, in general, is an energy-driven process. ${ }^{1-3}$ This occurs with enthalpic changes in the thermodynamic system, in which the system size is ideally infinite. However, if the system is confined within a finite boundary, the boundary effects become nontrivial while the system passes through from one thermalized state to another. These are primarily associated with the change of entropy rather than change of enthalpy, since the boundary effects due to confinement give rise to an entropic potential in lower dimensions. In recent years, the concept of entropic potential has been widely used in several issues, ${ }^{4-14}$ such as entropic stochastic resonance and resonant activation. A popular description of the system in reduced dimensions in this context is Fick-Jacobs equation which is essentially a Smoluchowski equation with a free energy term replacing the potential energy of the particle. The focus of the present study is the dynamical transition associated with the shape change of the confinement of a stochastic system between two entropic states.

To place the discussion in perspective, we first consider the classical diffusive motion of a particle subjected to a constant force in the transverse direction in a two-dimensional enclosure. The shape of the confined space is changed from a bilobal to a unilobal form (see figure 1). This two-dimensional problem can be

\footnotetext{
\#Dedicated to Prof. N Sathyamurthy on his 60th birthday

*For correspondence
}

analysed in one-dimension with an effective potential which is entropic in origin. It is therefore possible to calculate the work done on the system for changing shape at a finite rate and the associated change in entropy. Traditionally, the total work performed reversibly on the system is equal to the Helmholtz free energy difference $\Delta F$ between initial and final configurations,

$$
W=\Delta F=F_{B}-F_{A} .
$$

Here $F_{A}\left(F_{B}\right)$ refers to equilibrium free energy of the system with the parameter held fixed at $A(B)$. By contrast, when the parameter is changed along a chosen path at a finite rate, i.e., the system is changed irreversibly; Jarzynski ${ }^{15}$ showed that

$$
\begin{gathered}
\langle\exp (-\beta W)\rangle=\exp (-\beta \Delta F) \\
\Delta F=-\beta^{-1} \ln \langle\exp (-\beta W)\rangle,
\end{gathered}
$$

where $\beta=1 / k_{B} T$ and $\langle\ldots>$ denote a canonical ensemble average. The averaging of the concerned quantity is done over the measurement of that quantity for all the trajectories of the ensemble, keeping the path and the rate at which the parameters are switched, unchanged. This relation is independent of the path and the switching rate. Using this equation one can extract equilibrium information $\Delta F$ from an ensemble of non-equilibrium measurements. ${ }^{15-18}$ In what follows, we consider the free energy change of the stochastic dynamical system for deformation of shape of the confinement from one configuration to another. Our aim is to explore whether there occurs any phase transition or crossover during this shape transition of the system. In 


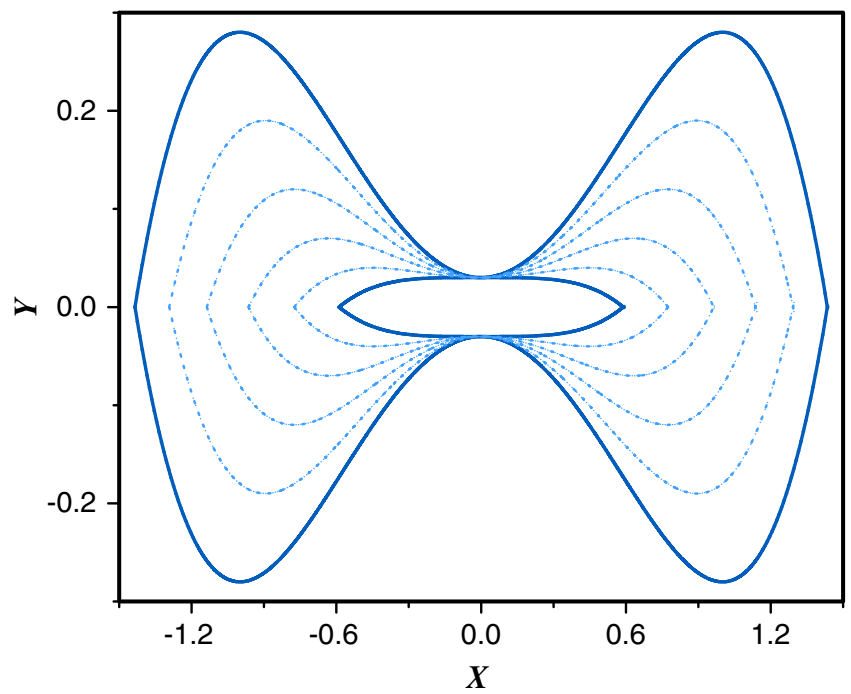

Figure 1. Temporal change in the shape of the confinement: Solid lines indicate boundary of initial and final states, dotted lines indicate boundary of intermediate states.

other words, can shape transition be realized as entropic phase transition?

The paper is organized as follows: In section 2 we discuss the model for overdamped Brownian motion of a particle in a two-dimensional confined space and evaluate the problem of entropic dynamical transition in terms of the change in shape and size of the confinement. In section 3 we map the problem in a reduced dimension and implement the Jarzynski relation. The analytical results are compared with numerical simulation in two dimensions in section 4. The paper is concluded in section 5 .

\section{Model description}

We consider the overdamped motion of a Brownian particle in a constrained geometry, subjected to a constant force $G$ in the transversal direction in terms of the following Langevin equation;

$$
\gamma \frac{\partial \vec{r}}{\partial t}=-G \overrightarrow{e_{y}}+\sqrt{\gamma k_{B} T} \zeta(t) .
$$

Here $\vec{r}$ denotes the position vector of the particle, $\gamma$ is the friction coefficient, $\overrightarrow{e_{x}}, \overrightarrow{e_{y}}$ are the unit vectors along $x$ and $y$ directions, respectively, and $\overrightarrow{\zeta(t)}$ is a Gaussian white noise with zero mean and obeys the fluctuationdissipation relation, i.e., $\left\langle\zeta_{i}(t) \zeta_{j}\left(t^{\prime}\right)\right\rangle=2 \delta_{i j} \delta\left(t-t^{\prime}\right)$, for $i, j=x, y . \mathrm{kB}$ is the Boltzmann constant and $T$ stands for absolute temperature. The symmetric structure of a two-dimensional system, confining the Brownian particles, is defined by a quartic double-well function,

$$
\omega_{l}(x)=L_{y}\left(x / L_{x}\right)^{4}-2 L_{y}\left(x / L_{x}\right)^{2}-b / 2=-\omega_{u}(x) .
$$

The expression (4) involves the geometrical parameters $L_{x}, L_{y}$ and $b$. Here $x$ and $y$ are position coordinates and $\omega_{l}(x), \omega_{u}(x)$ correspond to the lower and the upper boundary function of the system. $L_{x}$ corresponds to the distance between the bottleneck position and the position of maximal width of the enclosure, $L_{y}$ refers to the narrowing of the boundary function, and $b$ to the remaining width at the bottleneck (cf figure 2).

Consequently, $\omega(x)=\frac{\omega_{u}(x)-\omega_{l}(x)}{2}$ gives the local halfwidth of the enclosure. For the sake of dimensionless description, we scale all lengths by characteristic length $L_{x}$, i.e., $\tilde{x}=x / L_{x}, \tilde{y}=y / L_{x}$, which implies $\tilde{b}=b / L_{x}$ and $\widetilde{\omega}_{l}(x)=\omega_{l}(x) / L_{x}=-\widetilde{\omega}_{u}(x)$, time by $\tau=\left(\gamma L_{x}^{2} / k_{B} T_{R}\right)$, the corresponding characteristic diffusion time at an arbitrary, but irrelevant reference temperature $T_{R}$, i.e. $\tilde{t}=t / \tau$. In the rest of our discussion we shall omit the tilde symbols for clarity. In dimensionless form the Langevin equation and the expression for local half-width become,

$$
\begin{gathered}
\frac{\partial \vec{r}}{\partial t}=-G \overrightarrow{e_{y}}+\sqrt{D} \zeta(t) \\
\omega(x)=-\varepsilon x^{4}+2 \varepsilon x^{2}+b / 2,
\end{gathered}
$$

respectively, where the aspect ratio $\varepsilon=L_{y} / L_{x}$ and the dimensionless temperature $D=T / T_{R}$ are defined.

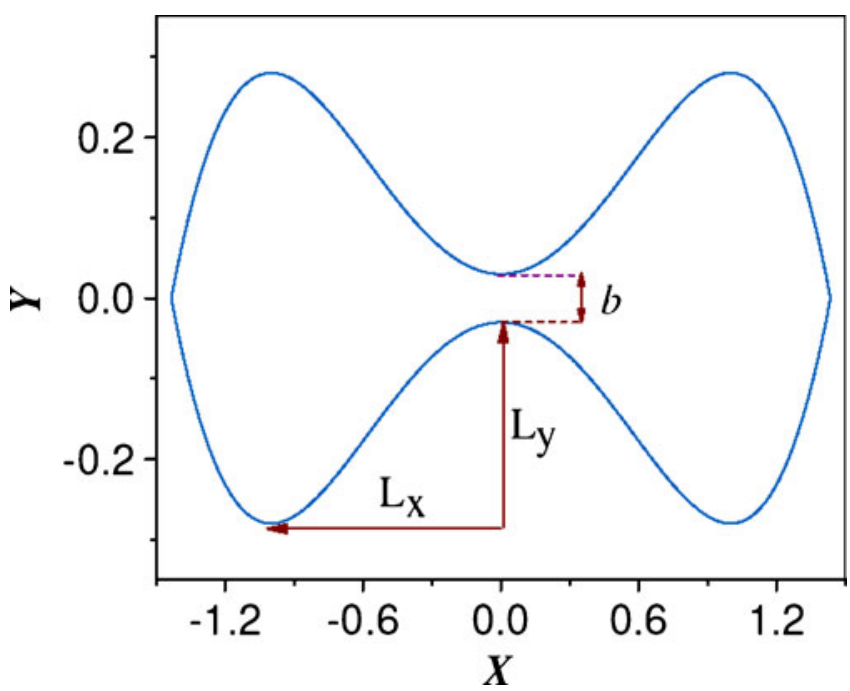

Figure 2. Shape of the system as described by equation 4 with its geometrical parameters. 
We now calculate the change in Helmholtz free energy difference between two equilibrium states of a system using Jarzynski equality (i.e., by calculation of irreversible work done) and the same by the relation obtained from equilibrium statistical mechanics, $\Delta F=-\beta^{-1} \ln \left(Z_{f} / Z_{i}\right)$, where $Z_{i}$ and $Z_{f}$ are the partition functions of the initial state and final state, respectively.

To calculate the irreversible work we need to introduce a parameter which monitors the system in going from an initial state to a final state. Let this switching parameter be $\lambda(t)$, which is incorporated in the equation of the local half-width of the system. This imposes a temporal dependency of the local half-width of the system,

$$
\omega(x, t)=-\varepsilon x^{4}+2\left(\varepsilon-\varepsilon_{0} \lambda(t)\right) x^{2}+b / 2 .
$$

Now, let us choose $\varepsilon_{0}=\varepsilon$ and $\lambda(t)$ varies from 0 to 1 during the time from 0 to $t_{f}$ such that the rate of change of $\lambda$ with time is a constant, i.e., $\dot{\lambda}=1 / t_{f}$.

At time $t=0, \lambda_{\text {initial }}=0$ then the equation of the local half-width takes the form,

$$
\omega(x, 0)=-\varepsilon x^{4}+2 \varepsilon x^{2}+b / 2 .
$$

Again, at time $t=t_{\mathrm{f}}, \lambda_{\text {final }}=1$, the equation of the local half-width is given by,

$$
\omega\left(x, t_{f}\right)=-\varepsilon x^{4}+b / 2 .
$$

The shape and size of the enclosure are depicted in figure 1 . The outermost and the innermost boundaries of the enclosure are shown as the initial and the final states. In-between these two equilibrium states the system adopts various shapes and sizes (for $0<t<t_{f}$ ). A few intermediate shapes of the system have been illustrated in figure 1.

\section{Reduction to one-dimension and the effective potential}

The diffusive dynamics in two dimensions as given by equation 3 with reflecting boundary conditions is difficult to solve analytically. The problem, however, can be greatly simplified by assuming a local equilibrium with a marginal distribution in the y-direction and thereby reducing the dimensionality of the problem. This reduction of dimension was first introduced by Zwanzig ${ }^{5,6}$ in a problem of diffusion in a narrow tube with varying cross section which was studied extensively by a number of groups over the last decade. ${ }^{7-14}$ The dyna- mics is described by the following two-dimensional Smoluchowski equation, ${ }^{19}$

$$
\begin{aligned}
\frac{\partial P(x, y, t)}{\partial t}= & D \frac{\partial}{\partial x} e^{-U(x, y) / D} \frac{\partial}{\partial x} e^{U(x, y) / D} P(x, y, t) \\
& +D \frac{\partial}{\partial y} e^{-U(x, y) / D} \frac{\partial}{\partial y} e^{U(x, y) / D} P(x, y, t),
\end{aligned}
$$

with reflecting boundary conditions at the confining walls. Here $P(x, y, t)$ is a probability distribution function, i.e., the probability of finding the particle at the point $(x, y)$ in a two-dimensional space at time $t$, and the potential function is given by $U(x, y)=G y$. We now introduce the marginal probability density $P(x, t)$ which is obtained by integration over the transverse coordinate;

$$
P(x, t)=\int d y P(x, y, t) .
$$

Integration of equation 10 over the transverse direction gives,

$$
\begin{aligned}
\frac{\partial}{\partial t} & \int d y P(x, y, t) \\
& =D \frac{\partial}{\partial x} \int\left(e^{-U(x, y) / D} \frac{\partial}{\partial x} e^{U(x, y) / D} P(x, y, t)\right) d y .
\end{aligned}
$$

Assuming local equilibrium in the y-direction, an $\mathrm{x}$ dependent effective energy function $V(x, t)$ (omitting irrelevant constants) is defined as,

$$
e^{-V(x, t) / D}=\int e^{-U(x, y, t) / D} d y .
$$

Now,

$$
\int \rho(y ; x) d y=1
$$

where, $\rho(y ; x)$ is the conditional local equilibrium distribution of $y$ at a given $x$. This is given by

$$
\rho(y ; x)=e^{-U(x, y) / D} e^{V(x) / D}
$$

which is normalized for every $x$. As a result, the twodimensional probability distribution can be approximately expressed as,

$$
P(x, y, t) \cong P(x, t) \rho(y ; x)
$$

and the equation for the marginal probability density becomes

$$
\frac{\partial}{\partial t} P(x, t)=D \frac{\partial}{\partial x} e^{-V(x) / D} \frac{\partial}{\partial x} e^{V(x) / D} P(x, t) .
$$

This is the Smoluchowski equation for an effective potential in one-dimension. 
From equation 13 the following relation follows:

$$
V(x, t)=-D \ln \left[\int_{\omega_{l}(x, t)}^{\omega_{u}(x, t)} e^{-U(x, y, t) / D} d y\right] .
$$

For a constant force $G$ in the negative y-direction, the effective potential function $V(x, t)$ becomes,

$$
V(x, t)=-D \ln \left[\frac{2 D}{G} \sinh \left(\frac{G \omega(x, t)}{D}\right)\right] .
$$

For an entropy-driven situation, $G \omega(x) / D<<1$, we have

$$
V(x, t)=-D \ln [2 \omega(x, t)] .
$$

Equation 17 is a time-dependent effective potential which is dominated by the entropy, along $\mathrm{x}$-direction in reduced dimension. As a result the potential of the system changes from its initial form to the final form as a function of time.

The temporal evolution of the effective potential is shown in figure 3. It is apparent that the potential is bistable in nature initially and gradually becomes a monostable one in the final state. From this analysis it follows that, the particles which were moving freely in a two-dimensional confinement, feels a spatiotemporal entropic potential due to reduction of dimensionality. The Langevin equation corresponding to the Fokker-Planck equation (equation 15) is given by

$$
\gamma \dot{x}=-V \prime(x, t)+F(t) .
$$

Here the Langevin force $(F(t))$ of the bath is modeled by a Gaussian, zero mean and delta correlated white noise, i.e., $\langle F(t)\rangle=0$ and $\left\langle F(t) F\left({ }^{t}\right)\right\rangle=2 D \delta\left(t-t^{\prime}\right)$,

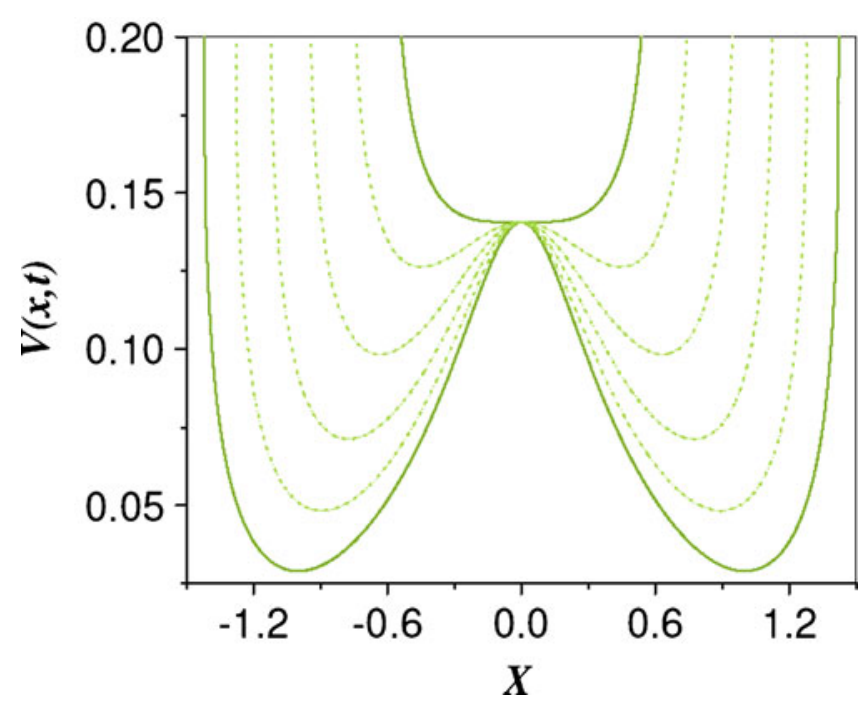

Figure 3. Time evolution of the entropic potential in the reduced dimension. Solid lines indicate the effective potential of final and initial states, and dotted lines indicate that of the intermediate states. Parameter set chosen: $b=0.06, \varepsilon=$ $0.25, \varepsilon_{0}=0.25$. where $D$ is the strength of the noise, i.e., $D=\gamma k_{B} T$. For the sake of simplicity we consider dissipation constant $(\gamma)$ as unity for the rest of the treatment. Furthermore the use of explicit form of $V^{\prime}(x, t)$ in equation 18 yields

$$
\dot{x}=D \frac{\left[8\left(\varepsilon-\varepsilon_{0} \lambda(t)\right) x-8 \varepsilon x^{3}\right]}{\left[-2 \varepsilon x^{4}+4\left(\varepsilon-\varepsilon_{0} \lambda(t)\right) x^{2}+b\right]}+F(t) .
$$

To follow the temporal evolution of the work done $(W)$ associated with the change of state we now introduce an appropriate equation for $\dot{W}$.

The time evolution of the work done is given by

$$
\dot{W}(x, t)=\dot{V}(x, t)=\frac{\partial V(x, t)}{\partial t} .
$$

For our case, $V(x, t)$ is a function of $\lambda(t)$. So, equation 20 can be rewritten as,

$$
\begin{aligned}
\dot{W}(x, t) & =\frac{\partial \lambda}{\partial t} \cdot \frac{\partial V(x, t)}{\partial \lambda} \\
& =\frac{2 D \varepsilon_{0} x^{2}}{t_{f}\left[-\varepsilon x^{4}+2\left(\varepsilon-\varepsilon_{0} \lambda(t)\right) x^{2}+b / 2\right]} .
\end{aligned}
$$

By solving equations 19 and 21 simultaneously we can evaluate the evolution of $x$ and $W$ with time. Having determined $W\left(t_{f}\right)$, the work done to transform the system from the initial state to the final state, we perform a canonical ensemble averaging of $\exp \left(-\beta W\left(t_{f}\right)\right)$. Making use of Jarzynski equality (equation 2) we calculate the free energy change for the transformation carried out in an irreversible pathway. Now, since both the initial and final states are in equilibrium, the partition functions ${ }^{1}$ of these states are given by,

$$
\begin{aligned}
Z_{i}= & \int_{-\infty}^{\infty} d p d x \\
& \times \exp \left[-\frac{\left(p^{2} / 2\right)-D \ln \left[2\left(-\varepsilon x^{4}+2 \varepsilon x^{2}+b / 2\right)\right]}{k_{B} T}\right], \\
Z_{f}= & \int_{-\infty}^{\infty} d p d x \\
& \quad \times \exp \left[-\frac{\left(p^{2} / 2\right)-D \ln \left[2\left(-\varepsilon x^{4}+b / 2\right)\right]}{k_{B} T}\right]
\end{aligned}
$$

So finally we can get the equilibrium free energy change using the equation,

$$
\Delta F=-k_{B} T \ln \left(Z_{f} / Z_{i}\right) .
$$

\section{Numerical simulations; calculation of work and free energy}

We numerically solve equations 19 and 21 for $x$ and $W$ using improved Euler method with reflecting 
boundary condition on the walls of the enclosure. The Gaussian white noise is generated using Box-Müller algorithm. For the entire calculation the values of $\varepsilon$ and $b$ are taken as 0.25 and 0.06 , respectively. The time step $(\Delta t)$ used is $10^{-3}$. The statistical averaging is carried out over 30000 to 40000 realizations of the trajectories. The linear coefficient $\varepsilon_{0}$, a constant parameter of the system, governs the nature of shape of the enclosure as well as the confined area indicating the 'phase' in which the free energy function is analytical and continuous.

In figure 4 we compare the numerically simulated variation of $\Delta F$ with $\varepsilon$ with the analytical results for several values of dimensionless temperature. $\varepsilon_{0}=0$, refers to a no change situation between the initial and the final states, rightly associated with a zero value of $\Delta F$. A gradual increase in $\varepsilon_{0}$ results in an increase of $\Delta F$ in a sigmoidal manner. From these plots it is found that all the numerical results are in good agreement with the analytical predictions.

From figure 4, it is evident that the change of free energy is greater for higher temperature for a particular value of $\varepsilon_{0}$. Since a dynamical transition is associated with the crossing of boundary between the two regions, it is necessary to look for any discontinuity or irregularity of the first and second derivatives of the free energy change with control parameter at the boundary. With this in mind, we plot the variation of the first derivative of the change in free energy $\left(\frac{\partial \Delta F}{\partial \varepsilon_{0}}=\psi\right.$, say) with $\varepsilon_{0}$ in figure 5a, b.

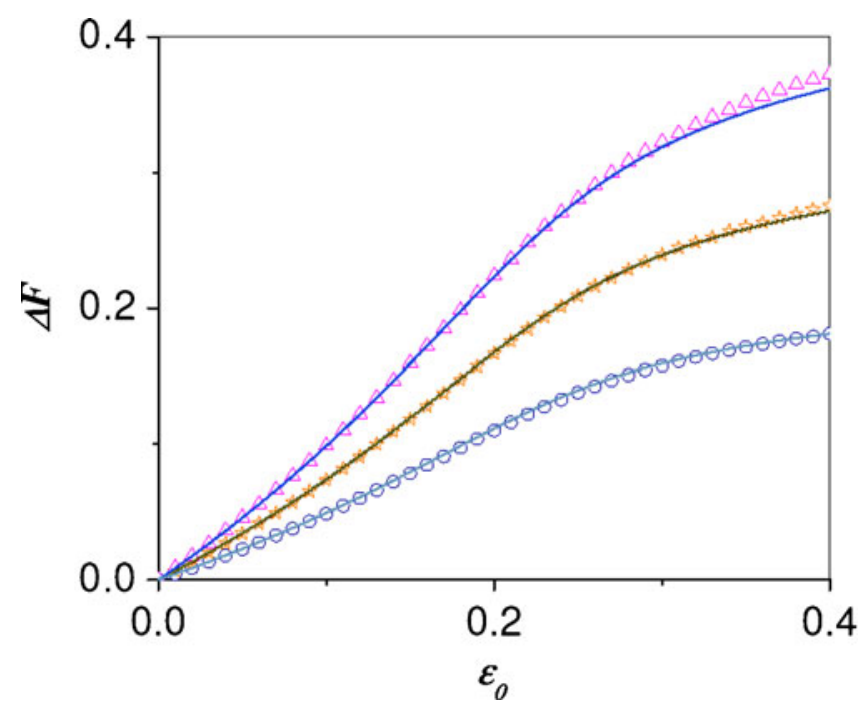

Figure 4. Plot of free energy change $(\Delta F)$ with $\varepsilon_{0}$ for different temperatures. Switching rate of the process is taken as $3.3 \times 10^{-2}$ for all cases. The points represent the numerical results and the lines denote the analytical findings for $k_{B} T=$ $0.100(\triangle) ; 0.075(\Delta) ; 0.050($ o), respectively.
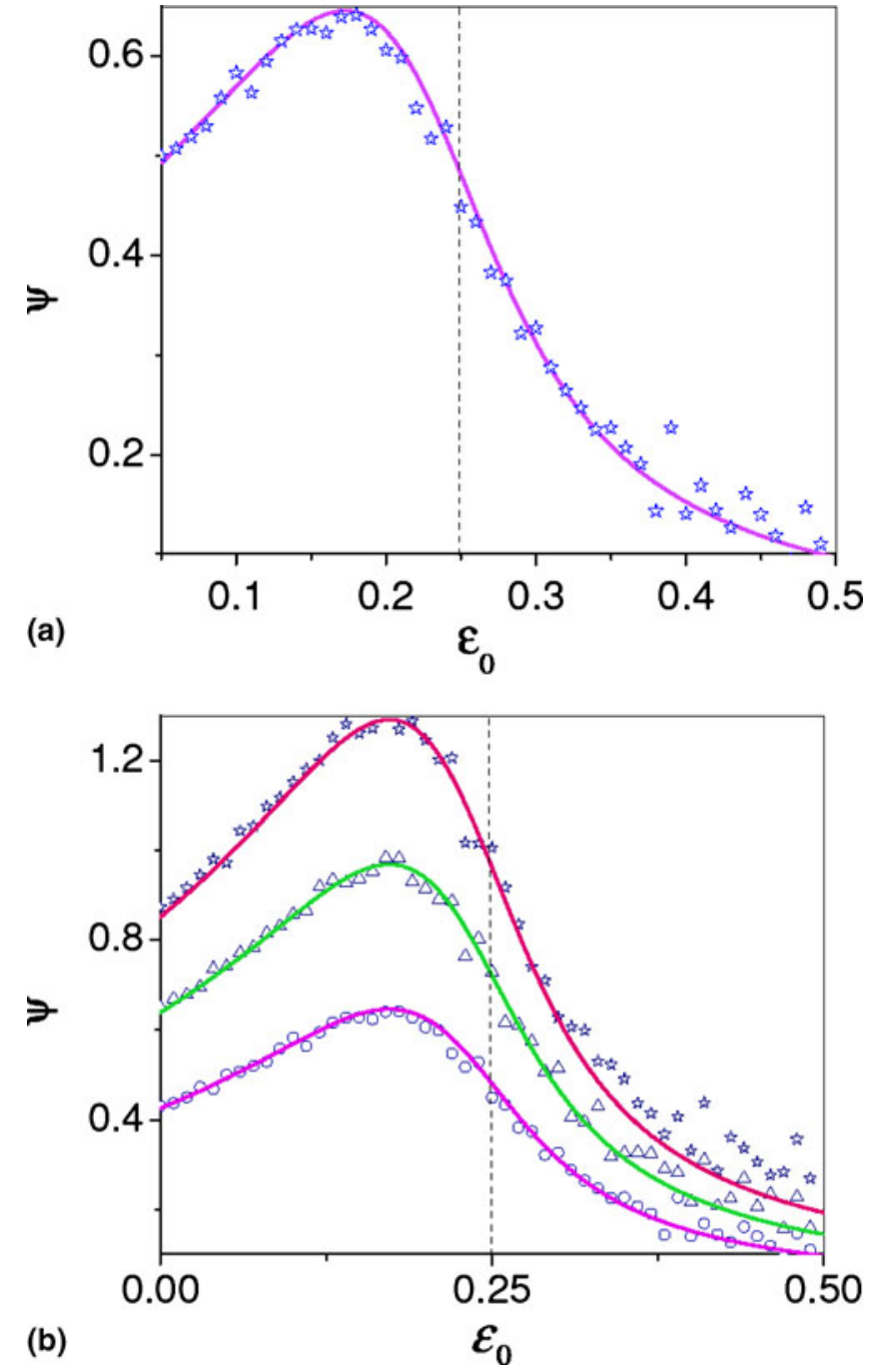

Figure 5. (a) A plot of $\psi$ versus $\varepsilon_{0}$ at $k_{B} T=0.05$. (b) The same plot but for three different temperatures $\left(\left[k_{B} T=0.100\right.\right.$ $(\bowtie) ; 0.075(\triangle) ; 0.050(\circ)])$. For all cases the switching rate is taken as $3.3 \times 10^{-2}$ and the points represent the numerical results and the corresponding lines denote the analytical results.

Now in the spirit of the traditional way of deriving thermodynamic information and characterizing equilibrium phase transition, one may define an extensive variable, an order parameter type quantity as, $\left(\frac{\partial \Delta F}{\partial \varepsilon_{0}}\right)_{T}$. From figure $5 \mathrm{a}$ it is observed that there is a sharp inflection in the first derivative of the free energy change. The order parameter gradually increases to a maximum followed by a sharp fall at $\varepsilon_{0}=\varepsilon$. The variation of the order parameter with $\varepsilon_{0}$ illustrates two types of dynamical transitions. Our observations can be summarized as follows.

(i) The first one corresponds to a transition from an ordered state to a disordered state. This is due to the fact that, in this temperature domain, the 
distribution of the kinetic energy gains a broad range. The kinetic energy of particles is of the order of $k_{B} T$ and in our case the potential is directly proportional to $k_{B} T$. As a result the two effects compensate each other. So, the maximum of the first-derivative does not shift for different temperatures. This is clear from figure $5 b$.

(ii) The second type of transition is due to a change of distribution function. This is reflected in a very sharp change in the order parameter at $\varepsilon_{0}=\varepsilon$, as shown in figure $5 \mathrm{~b}$.

It is also instructive to plot the negative of second derivative of the free energy change $\left(\frac{\partial^{2} \Delta F}{\partial \varepsilon_{0}^{2}}=-\phi\right.$, say), as a function of $\varepsilon_{0}$. This is depicted in figure 6 . The plot shows a maximum at $\varepsilon_{0}=\varepsilon$, supporting the fact that the inflection point of figure $5 \mathrm{~b}$ lies at the same point.

The principal finding of our study is the sharp inflection point at $\varepsilon_{0}=\varepsilon$, found in figure $5 \mathrm{a}, \mathrm{b}$. This indicates that at $\varepsilon_{0}=\varepsilon$ as the shape of the enclosure changes from bi-lobal to a unilobal one, the particles inside the confinement experience a sudden change in nature of the effective force field. It is worth-mentioning that this crossover occurs in isothermal condition and thus only the change in shape of the system is responsible for such a free energy difference. The change in shape of the enclosure is reflected in the change in effective entropic potential from bi-stable to mono-stable form. At $\varepsilon_{0}=$ $\varepsilon$, we expect a phase transition between the two thermalized states. That the transition described is due to a change in shape from one initial volume to another can be ascertained by studying the free energy change for

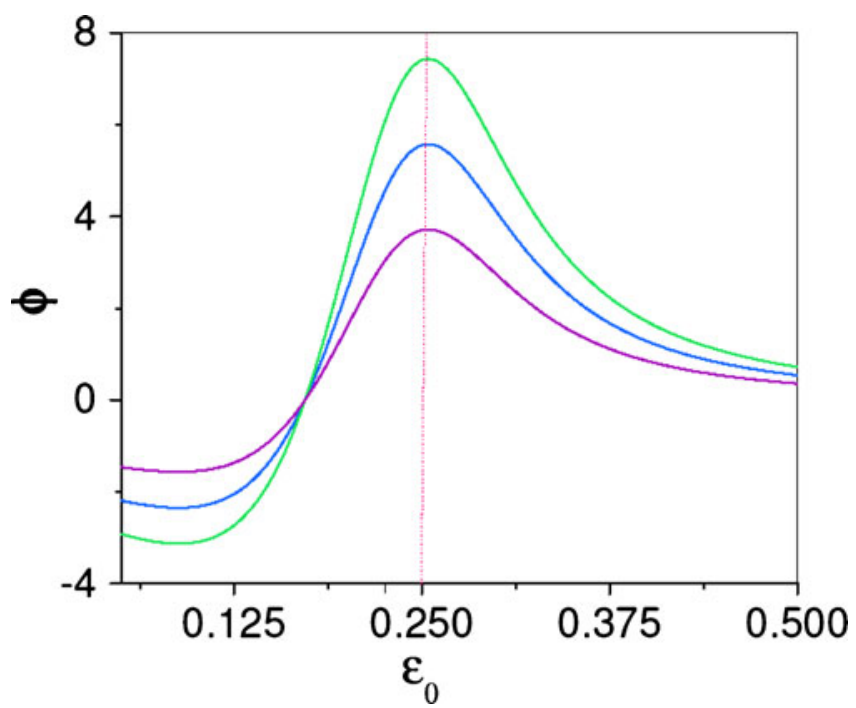

Figure 6. Plot (analytical) of $\phi$ versus $\varepsilon_{0}$ for $\left(k_{B} T\right)=$ 0.100 (green), 0.075 (blue), 0.050 (violet).
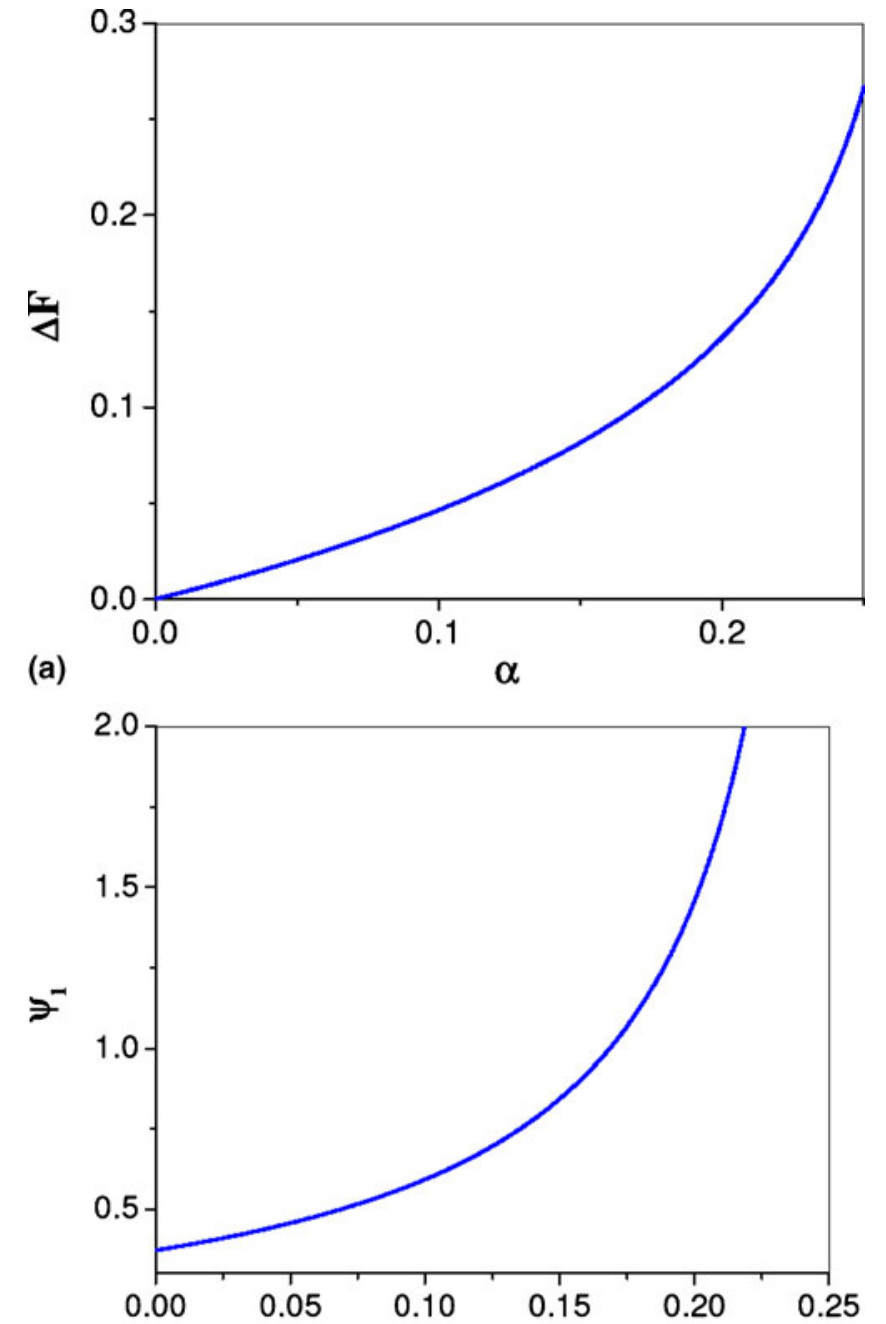

(b)

$\alpha$

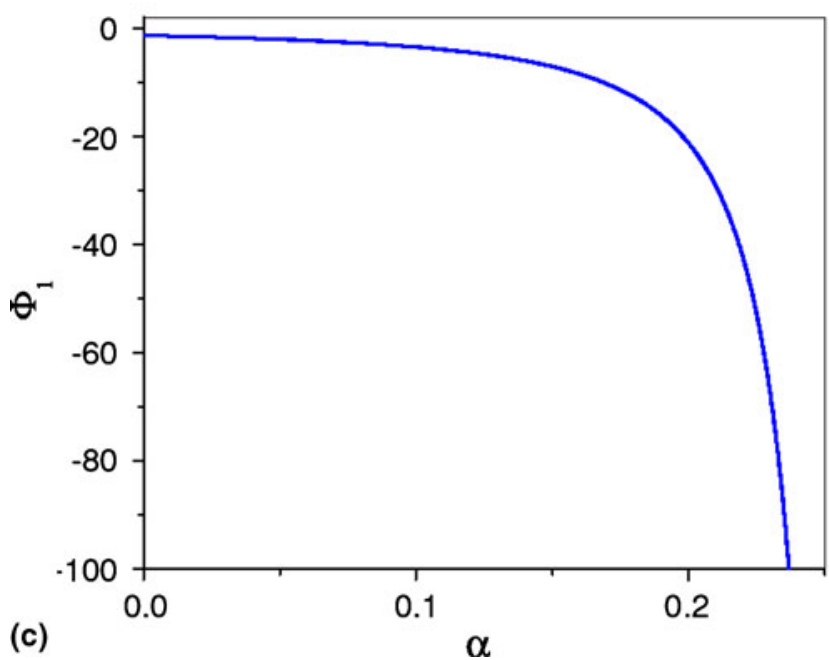

Figure 7. (a) Analytical plot of free energy $(\Delta F)$ change against $\alpha$. (b) Analytical plot of $\Psi_{1}=\frac{d \Delta F}{d \alpha}$ against $\alpha$. (c) Analytical plot $\Phi_{1}=-\frac{d^{2} \Delta F}{d \alpha^{2}}$ against $\alpha . k_{B} T$ is taken as 0.05 in all cases. 
the change in volume of the system from the same initial value keeping the shape of the system intact. To this end let us define an enclosure with the local half-width, described by the following equation,

$$
\omega(x, t)=-\alpha(1-\lambda(t))\left(\varepsilon x^{4}-2 \varepsilon x^{2}-b / 2\right),
$$

where $\alpha$ is an external parameter controlling the area of the enclosure and $\lambda(t)=0$ and 1 stand for the initial $(t=0)$ and final states $\left(t=t_{f}\right)$, respectively. We then record the isothermal free energy change between the states by analytically calculating the partition function of the related states.

To explore the nature of the free energy change associated with the change of state as described by equation 25 , we have plotted $\Delta F$ against the system parameter $\alpha$. This has been shown in figure 7a.

The plot of the variation of the first derivative of free energy change $\left(\frac{\partial \Delta F}{\partial \alpha}=\psi_{1}\right)$ as a function of $\alpha$ gives a monotonous curve (figure $7 \mathrm{~b}$ ). No inflection is observed in this case, i.e., we do not find any signature of phase transition. We have also plotted the negative of the second derivative of free energy change $\left(\frac{\partial^{2} \Delta F}{\partial \alpha^{2}}=-\phi_{1}\right)$ against $\alpha$. This plot is also continuous without any extremum (figure $7 \mathrm{c}$ ). All these figures $(7 \mathrm{a}-7 \mathrm{c})$ support the fact that the phase transition occurs due to the change of shape of the system.

\section{Conclusion}

It is well known that in case of liquid-gas phase transition, Gibbs free energy vs volume plot shows two minima below the critical temperature, whereas it has a single minimum at the critical point. For our case, the entropic potential is bistable for $\varepsilon_{0}<\varepsilon$, and it transforms to a monostable one at $\varepsilon_{0}=\varepsilon$. Extrapolating the analogy with the liquid-gas phase transition, one can define $\varepsilon$ as the critical point for our case. We have defined $\frac{\partial \Delta F}{\partial \varepsilon_{0}}$ as the order parameter. From the plot of $\frac{\partial \Delta F}{\partial \varepsilon_{0}}$ vs $\varepsilon_{0}$, it is clear that a divergent fall of the order parameter is observed around $\varepsilon_{0}=\varepsilon$ when the potential transforms into a monostable form from the bistable one at the critical point. The sharp change of the order parameter gives a signature of phase transition at the critical point. We may therefore conclude that shape transition bears a close kinship to entropic second order phase transition.

Lastly, it is also pertinent to mention that the phenomenon does not possess the characteristics of noiseinduced transition of energetic ${ }^{20-22}$ or entropic ${ }^{23}$ origin where the character of stationary probability distribution changes with the variation of the strength of external multiplicative noise acting on the particles trapped in a bistable potential well or in a bilobal confinement, respectively and is thus not to be confused with it. The dynamical transition associated with the change of shape of the confinement is generically different from these cases. We believe that further studies in this direction will throw more light into the nature of entropic potential.

\section{Acknowledgements}

Thanks are due to $\mathrm{S}$ Adhikari for providing computer facility under the scheme SR/S1/PC/13/2008, Government of India. M. Das would like to thank the Council of Scientific and Industrial Research, Government of India for partial financial support.

\section{References}

1. Callen H B 1985 Thermodynamics and an introduction to thermostatistics (New York: Wiley) 2nd Ed.

2. Ma S K 1976 Modern theory of critical phenomena (Benjamin, Reading, MA)

3. Stanley H E 1971 Introduction to phase transitions and critical phenomena (Oxford)

4. Jacobs M H 1967 Diffusion processes (New York: Springer)

5. Zwanzig R 1992 J. Phys. Chem. 963926

6. Zwanzig R 1983 Physica 117A 277

7. Reguera D and Rubi J M 2001 Phys. Rev. E 64 061106

8. Reguera D, Schmid G, Burada P S, Rubi J M, Reimann P and Hänggi P 2006 Phys. Rev. Lett. 96130603

9. Burada P S, Schmid G, Reguera D, Rubi J M and Hänggi P 2007 Phys. Rev. E 75051111

10. Burada P S, Schmid G, Reguera D, Vainstein M H, Rubi J M, Hänggi P 2008 Phys. Rev. Lett. 101130602

11. (a) Mondal D, Das M and Ray D S 2010 J. Chem. Phys. 132 224102; (b) Mondal D and Ray D S 2010 Phys. Rev. E 82032103

12. Hänggi P, Marchesoni F and Nori F 2005 Ann. Phys. 14 51

13. (a) Ghosh P K, Marchesoni F, Savel'ev S E and Nori F 2010 Phys. Rev. Lett. 104 020601; (b) Ghosh P K and Marchesoni F 2010 Eur. Phys. Spec. Top. 18741

14. Heinsalu E, Patriarca M and Marchesoni F 2009 Eur. Phys. J. B 6919

15. (a) Jarzynski C 1997 Phys. Rev. Lett. 78 2690; (b) 2004 J. Stat. Mech.: Theory Exp. P009005

16. Jarzynski C 1997 Phys. Rev. E 565018

17. Crooks G E 1999 Phys. Rev. E 602721

18. (a) Ghosh P K and Ray D S 2008 Physica A 387 6443; (b) Van den Broeck C, Parrondo J M R and Toral R 1994 Phys. Rev. Lett. 733395 
19. Risken H 1989 The Fokker-Planck equation (Berlin: Springer) 2nd Ed.

20. (a) Horsthemke $\mathrm{W}$ and Lefevere R 1977 Phys. Lett. A 64 19; (b) Schenzle A and Brand H 1979 Phys. Rev. A 201628

21. (a) Horsthemke W and Lefevre R 1983 Noise-induced transitions. Theory and application in physics, chemistry and biology (Berlin: Springer); (b) García-Ojalvo J and
Sancho J M 1999 Noise in spatially extended systems (New York: Springer-Verlag)

22. (a) Gammaitoni L, Marchesoni F, Menichella-Saetta E and Santucci S 1993 Phys. Rev. E 49 4878; (b) Luo X and Zhu S 2003 Phys. Rev. E 67021104

23. Mondal D, Das M and Ray D S 2010 J. Chem. Phys. 132 224102 